\title{
XVII. On a new theory of the tides
}

\section{Capt. Walter Forman}

To cite this article: Capt. Walter Forman (1822) XVII. On a new theory of the tides, Philosophical Magazine Series 1, 60:292, 88-93, DOI: 10.1080/14786442208652798

To link to this article: http://dx.doi.org/10.1080/14786442208652798

曲 Published online: 29 Jul 2009.

Submit your article to this journal

Џ Article views: 3

Q View related articles $₫$ 
observatory for instance) where the complication attending the other would render it quite inapplicable.

5. If we use the numbers formerly noticed for $a$ and $\lambda$, and put $w=2.3$ troy grains; then $\frac{48 a}{\lambda}=0233$, or $\frac{1}{50}$ nearly; and $\frac{48 a w}{\lambda}=26$, or $\frac{1}{4}$ nearly; and the formulæ (2) and (3) will become

$$
\begin{aligned}
& x=\rho-\frac{8 x}{40}, \\
& y=m-\frac{8 x}{4} .
\end{aligned}
$$

These expressions are very simple and easily remembered. If to the scale of the evaporating thermometer another scale were attached, showing the maximum tensions at the various degrees of temperature; or one showing the maximum quantities of humidity in a cubic foot at the same temperatures; we should only have to deduct $\frac{1}{40}$ of the observed depression to have the actual tension sought, or $\frac{1}{4}$ of the same quantity, to have the actual quantity of vapour in a cubic foot. As $\delta \tau$ will always be confined to a few degrees, a small table containing the quantities to be subtracted might be engraved apart on the scale of the instrument.

The purpose of these observations is to explain the method proposed by Dr. Hutton for finding the moisture of the atmosphere on its own principles, and to disengage it from all mechanism foreign to it. The process requires nothing more than two thermometers which we have supposed to be constructed according to the centigrade scale. It is greatly to be wished that a number of experiments were made sufficient for ascertaining the practical fitness of the method for accomplishing its end.

J. IVoRY.

XVII. On a Nere Theory of the Tides. By Capt. Forman, of the Royal Navy.

To the Editors of the Philosophical Magazine and Journal.

Gentlemen, - $\mathbf{I}_{\mathrm{F}}$ the facts and arguments contained in the following letter are true, they must add to our stock of knowledge, and ought to be made known; if they are not, they can easily be refuted; and therefore, as in either case they can do no harm, I shall feel obliged if you will find room for them in your Magazine. I am, gentlemen, \&c.

W. Fonmana 


\section{To the Members of the Astronomical Society.}

Gentremen,-As you have thought it necessary, in a paper addressed to the public, to call the attention of astronomers to that branch of astronomy which is connected with the rising of the tides, it is evident, that in your opinion the old hypothesis is not quite satisfactory; or at least that you wish to be furnished, by those who have had the opportunities of witnessing their phænomena in different parts of the world, with such additional facts and documents as may either fully establish the old theory, or lead to the discovery of a better.

In obedience then to this call, I beg leave to submit to your judgement a Treatise on the Tides, which I have just written, and which, by taking in the compressibility of water as a cooperating principle, will account for several very important phænomena that cannot be explained in any other way. Thus, for instance, if the compressibility of water be admitted in $a$ sufficient degree, as the moon's attraction (being in opposition to the earth's) must take off a portion of the gravity of every particle of water, these particles must necessarily expand in propertion to the weight that is taken off them; and, as the sum of the expansion of a great many particles must be a great deal more than the sum of the expansion of a few, we shall have a considerable rising in the deep parts of the ocean, without any sensible alteration in lakes and shallow water. Thus, then, if we admit the compressibility of water in a sufficient degree, we shall have a principle that will satisfactorily explain why the flood tide, in all places, always comes from the ocean; why there are no tides in lakes and some inland seas; why the tides do not rise to the same height in all places where the moon is equally vertical ; and lastly, why other loose substances are not raised by the power of the moon's attraction as well as water. But how can we account for all this, if we deny the compressibility, and consequently the expansion, of water?

If the moon's attraction, co-operating with the centrifugal force of bodies, is capable of raising the tides in the ocean, why does it not also raise the waters in lakes and ponds? and why are not all other loose substances affected in the same manner? If the earth, as was supposed by Newton, is constantly drawn towards the moon by the power of her attraction, and the waters on the same side, by being so much nearer than the central parts of the earth, are made to move so much faster, and thus are raised above their ordinary level, why are not all other loose substances, that are equally near, raised in the same manner? There is only one answer can be given to these questions. None of these substances are elastic; but Vol.60. No. 292. Aug. 1822. 
water is : and in proportion to its depth will be the degree of its expansion.

The compressibility of water to a certain extent has been proved by Mr. Perkins, and is now, I understand, admitted by all the philosophers. The only question then is, Whether it be compressible in a sufficient degree to produce the effects I have supposed. And, as no one can pretend to set bounds to the extreme depth of the ocean, or say to what degree water may be compressed in its deepest parts, no one can say that my hypothesis is impossible, or even improbable; while every one who is capable of thinking, when he considers that the moon's attraction has no power to lift the smallest or lightest substance that happens to be loose upon the surface of the earth, must be convinced that it could have no power to raise water, if it was not aided by some other principle; and surely there is no other principle that can aid in producing this effect, besides the elasticity of the particles of water.

Mr. Perkins, by a force which he considered equal to the weight of 320 atmospheres, or about two miles depth of water, compressed the water in a piezometer at the rate of three and a half per cent. In what manner the experiment was contrived I do not pretend to know; but, when we consider that water, in another experiment of his, forced its way into a botthe that was corked and sealed, it is certainly very possible that some of these minute particles might have escaped from the instrument before it was weighed, and thus the degree of compressibility may have been considerably underrated. 'This appears to me to be the more likely, because in another of Mr. Perkins's experiments the degree of compression was evidently a great deal more. In this experiment, the water, which had forced its way through the sealing of an empty porter bottle, at the depth of five hundred fathoms, on being drawn up and the external pressure in great measure diminished, expanded to such a degree that it forced the cork up against the coverings, compressed it into half its size, and then burst the bottle. As we have no means of measuring the degree of compression in this experiment, we nust content ourselves with a rough estimate; but it is evident that it must have been equal to the space that was occupied by the cork before it was forced up, because the bottle burst afterwards; and this, at the lowest calculation, could not have been less than three per cent. when the force applied was very little more than the weight of one hundred atmospheres. A quart bottle is generally supposed to hold about a dozen commonsized wine-glasses; and if we suppose the space that was occupied by that part of the cork that was forced upwards to be 
the fourth part of a wine glass, it will make the expansion of the water, before the bottle burst, to be about two per cent., or the fiftieth part of the whole. 'This however is but a small part of the expansion; for, as the water forced its way in through, or rather under, the coverings, we must suppose that some of it escaped in the same way; and yet the force of the expansion still continued to such a degree, that, when the cork could no longer give way, it burst the bottle. Here then we have positive proof that the expansion of water, at the depth of only five hundred fathoms, was at least more than two, and in all probability was as much as four or five, per cent.; and who will say that the extreme depth of the ocean may not extend to three, four, or even five hundred miles *, and that the compression of water at those depths may not exceed even a hundred per cent.? It is not too much to suppose that the average of the compression of water in the deepest parts of the ocean, may be at least twenty-five per cent.; and, if we take the depth of the ocean at two hundred milest, the expansion or rise of the waters at that depth, if the entire gravity of every particle was taken off, would amount to 50 miles or 48,000 fathoms; and if we divide this sum by 2400 , which I take to be the amount of the power of the moon's attraction, we shall have a rising of the waters in the deep parts of the ocean equal to the height of 20 fathoms, and which is fully sufficient to account for all the phænomena connected with the rising of the tides.

Whether or not this estimate will be admitted by philosophers is of very little moment, since I have it in my power to prove, by undeniable facts, that the expansion of water is the immediate cause of the rising of the tides; and I beg leave here, gentlemen, to request your particular attention to the facts I am about to produce, and the inference I shall deduce from them.

During the time of flood tide, when the waters are rising, instead of showing any disposition to go toreards the moon, they press downwards towards the earth's centre; which evidently proves that they are pushed upwards by the expansion of the particles below, and not pulled upwards by the power of the moon's attraction; for it is impossible to account for the tendency of the waters to press downwards, at the very moment that they are rising, without supposing a sufficient degree

* If we may believe some of the geological writers, the ocean has no bottom at all, and the earth is nothing more than a crust formed upon its surface. This is a doctrine, however, I do not subscribe to.

+ If we take the depth of the ocean at four hundred miles, a mean compression of twelve and a half per cent. will be sufficient for my purposc. 
of expansion in the particles below to push them upwards; and therefore, however it may appear to contradict our experiments and preconceived opinions, the fact is indisputable.

The same argument applies with equal force to the rising of the tides on the side opposite the moon; for, if the waters on this side were left behind, as Newton supposed, by the other parts of the earth being more strongly attracted by the moon in consequence of being so much nearer, or were carried upwards by a centrifugal force, they could have no tendency to press downwards; and yet we find that, at the very moment they are rising upwards, it is nothing but the resistance of the water below that prevents them from falling down towards the earth's centre. On this side then, as well as on the other, there must be an expansion of the particles of water to produce a rising of the tides; and, as the direction of the moon's attraction is here the same as the earth's, we can only account for this diminution of the weight or gravity of the particles of water on this side the earth, by supposing that the power of the moon's attraction takes off some portion of the power of the earth's attraction, in the same manner as $I$ have shown the attraction of one magnet will diminish the attraction of another, when their two ends are of the same denomination.

You see then, gentlemen, that this is not a question of mere opinion : it is a question of facts, and is to be proved, not by supposing what the known degree of the compressibility of water may be capable of producing, but what it actually does produce. In short, we have only to take a handful of water out of the ocean, at the time of the rising of the tides, to be convinced that the expansion of water is the immediate cause of the phænomenon; because, if the waters were pulled upward by the power of the moon's attraction and not pushed upwards by the expansion of the particles below, this water would not fall back to the earth, until the influence of the moon's attraction had gone off.

Here then, gentlemen, upon these grounds I rest my case; and I call upon you, and, as I intend that this letter shall go forth into the world, I call upon every astronomer and every philosopher, to answer the following questions: Do the waters at the time of their rising, press downwards, or do they not? If they do, how are we to account for their rising, except by supposing that they are pushed upwards by the expansion of the particles below? If you can account for this in any other way, I am ready to confess that, as my hypothesis, in that case, will not be wanted, you will effectually deprive me of the only grounds upon which I can make good its claim to supersede the old one. But if you cannot, you must acknowledge that 
the expansion of water is the immediate cause of the rising of the tides; and then, as philosophers, as lovers of truth, as men whose judgement will be rejudged by a succeeding generation, that will know nothing of the bias and prejudices of the present day; but, above all, as men of integrity and honour, you are bound to admit so much of my theory as depends upon the expansion of water*.

In speaking of the bias and prejudices of the present day, $I$ beg to be understood as merely meaning to infer, that opinions, which we have been taught in our infancy to revere as the most sacred truths, very frequently take so strong a hold upon the mind, that they are not easily eradicated even when they can be proved to be wrong; to venture to dispute them is little short of being guilty of a crime, and we shut our ears against every argument by which they can be oppugned. It occasionally happens, however, that the truth of some one of these dogmas, especially when it is grounded upon no better argument than authority, is called in question: this produces discussion, the struggles of its advocates hasten its downfall; and though we may still obstinately cling to it with the tenacious adherence of drowning men, it loses its hold upon posterity, and a succeeding generation, considering it merely in the light of a debatable question, judges of it by its intrinsic merits alone, and, throwing aside all prejudice, makes it give place to a better system. It is thus that opinions, which in the time of Galileo were held to be indisputable, are now universally exploded even by the most ignorant.

$$
\text { I am, gentlemen, }
$$

Your most obedient and very humble servant, Walter forman.

* Let us suppose a man to be carrying a weight upon his shoulders, and occasionally to be stooping down, and standing upright. Would not the continued pressure of the weight upon the man's shoulders, be a sufficient argument that it was not pulled upwards by an invisible power above, but was pushed upwards by his own exertions? and are not both cases precisely similar? If we could, by any possibility, take away the water below, can any one believe that the power of the moon's attraction could prevent the water above from falling down? and, if it could not, how can we account for the rising of the tides, except by supposing expansion in the particles of water?

XVIII. True apparent Right Ascension of Dr. Masketyne's 36 Stars for every Day in the Year 1822, at the Time of passing the Meridian of Greenwich. By the Rev.J.Grooвy.

[Continued from page 55 .] 